\title{
DETERMINAÇÃO EXPERIMENTAL DOS PARÂMETROS DE UM MODELO PARA UM PROCESSO MULTIVARIÁVEL DE QUATRO TANQUES ACOPLADOS.
}

\author{
M.C. de Freitas, F.V. da Silva. \\ Universidade Estadual de Campinas, Faculdade de Engenharia Química. \\ E-mail para contato: marianacarvalhodefreitas@hotmail.com
}

\begin{abstract}
RESUMO - Este trabalho teve como objetivo a determinação dos parâmetros de um modelo para a representação do comportamento dinâmico da variável nível em um protótipo experimental de quatro tanques acoplados. Inicialmente, foram determinadas as relações matemáticas entre a vazão e o percentual de variação da rotação das bombas do sistema. Com os resultados foi possível a determinação dos parâmetros do processo considerando-se um comportamento não-linear do sistema. Definido o modelo será possível realizar simulações para a determinação das estratégias de controle baseados em técnicas de inteligência artificial.
\end{abstract}

\section{INTRODUÇÃO}

O problema da modelagem e do controle de sistemas de tanques de armazenamento tem grande importância na indústria química moderna. Seu estudo é motivado tanto com o intuito de diminuir gastos com energia elétrica referente ao bombeamento de fluidos, quanto para aperfeiçoar a segurança de uma planta industrial.

Considerando-se o primeiro quesito, pode-se compreender, através de trabalhos como este, a importância da escolha e do uso adequado das válvulas, visto que dependendo da forma de uso, elas acarretam altas perdas de carga. Tais perdas muitas vezes não possuem comportamento linear com a potência utilizada nas bombas e a abertura da válvula, não sendo trivial achar os pontos ótimos para o processo. Quanto ao segundo quesito, sabe-se que certas substâncias exigem armazenamentos bastante específicos em termos de segurança. O software utilizado neste trabalho - Automation Studio (configurador do Controlador Lógico Programável utilizado no sistema)- permitiu o monitoramento on-line da variável nível dos tanques do sistema, além da programação de alarmes de segurança e intertravamentos (chaves de nível alto e Chaves de nível baixo virtuais).

Logo, o desenvolvimento de conhecimentos e práticas relativas ao controle e modelagem de tanques gera um retorno positivo para sistemas químicos, seja a nível industrial, seja a nível laboratorial.

\section{DESCRIÇÃO DO PROTÓTIPO EXPERIMENTAL}

O protótipo experimental do sistema multivariável de quatro tanques acoplados (Figura 1) está montado no Laboratório de Instrumentação e Redes Industriais da Faculdade de Engenharia Química da UNICAMP e é composto por: 


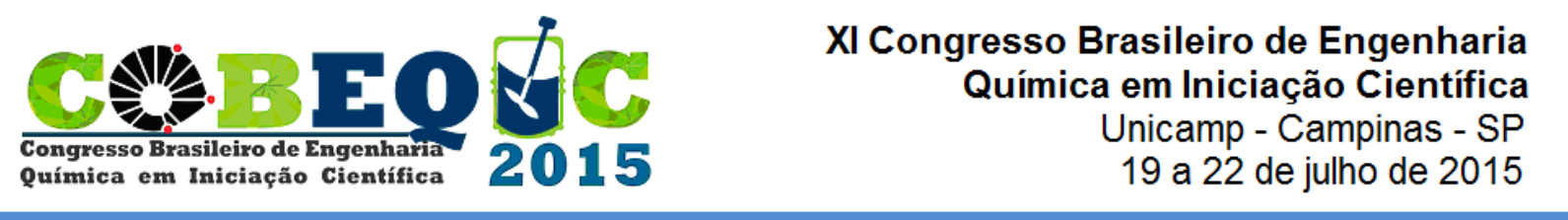

- 4 tanques iguais, de acrílico, diâmetro de 15,2 cm e altura de $80 \mathrm{~cm}$ com marcação de nível;

- 4 sensores piezoresistivos para medição do nível;

- 12 válvulas do tipo esfera (característica de abertura rápida);

- 2 bombas centrífugas trifásicas de $0,5 \mathrm{cv}$ cada, com inversores de frequência $(0-60 \mathrm{~Hz})$

A automação é composta por: Controlador Lógico Programável (B\&R, modelo PP45)

Figura1: (a) Protótipo Experimental do Sistema de Quatro tanques acoplados, (b) diagrama P\&ID do sistema.

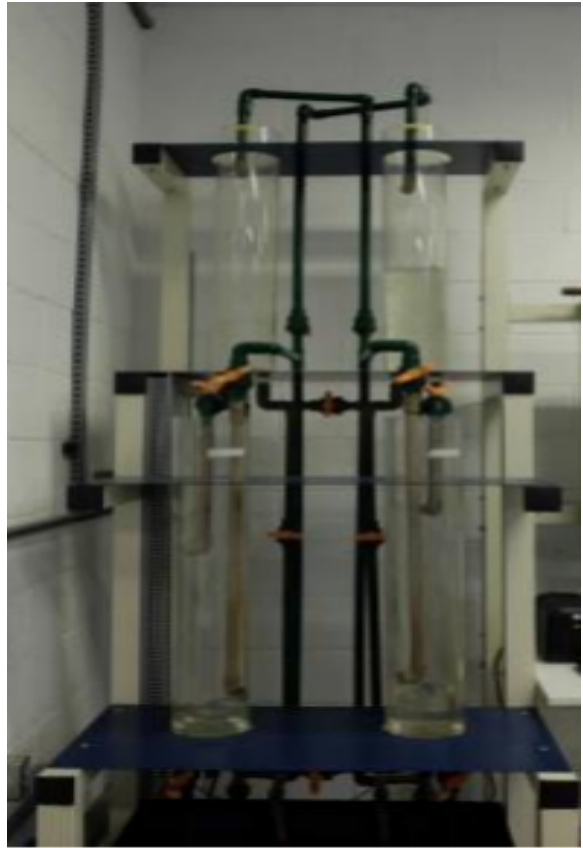

(a)

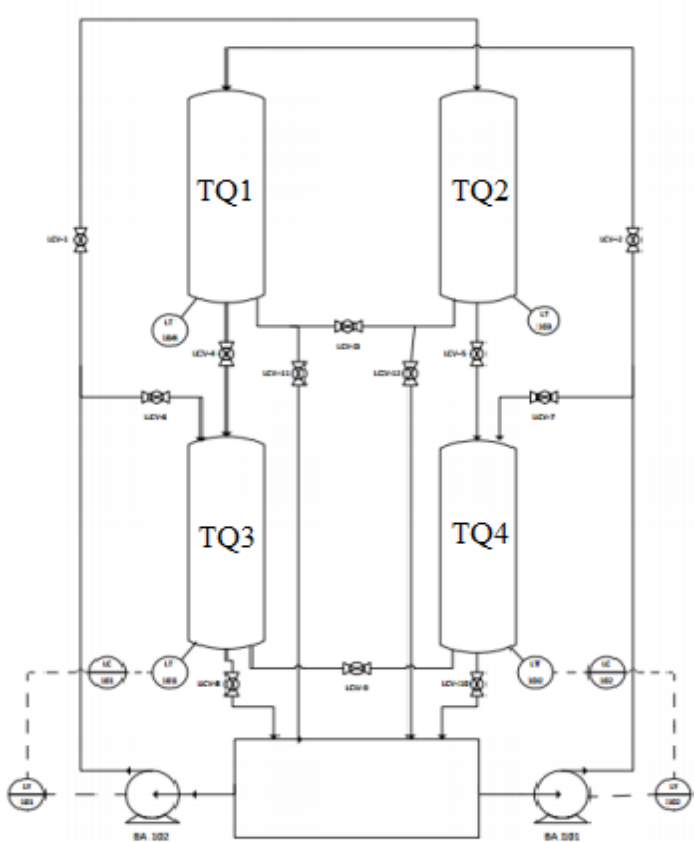

(b)

\section{DETERMINAÇÃO DAS VAZÕES DO SISTEMA}

\subsection{Calibração dos Sensores de Nível}

Inicialmente foi necessário realizar a calibração dos quatro sensores piezoresistivos para medição correta dos níveis dos tanques. A escala do nível foi definida como percentual (0 a $100 \%)$, sendo a altura máxima (100\%) igual a $60 \mathrm{~cm}$, equivalendo a um volume de controle de 11L. Foram colocadas marcadores visuais do níveis (0 a 100\%) nos tanques, e assim podese calibrar as variáveis do software de acordo com o valor da escala visual.

O valor da resistência elétrica lida por cada sensor é convertida em um sinal padrão de 4 a $20 \mathrm{~mA}$. Este sinal é convertido para um sinal binário recebido. 


\subsection{Determinação das Vazões e Níveis do Sistema}

As frequências de rotação (intrinsecamente relacionadas à potência fornecida) das número binário entre 0 e 32768 no CLP e enviado ao computador. No software do CLP foram definidas variáveis que realizavam o cálculo do volume de líquido no tanque em função do valor do

bombas são as variáveis manipuladas do sistema. Foi definido que as frequências variariam entre $0-60 \mathrm{~Hz}(0-100 \%)$.

O conjunto de válvulas do tipo esfera presentes no protótipo possui diferentes funções no sistema. Tem-se válvulas nas linhas que partem diretamente das bombas e servem para modular as vazões de entrada. O valor da vazão que passa por estas válvulas depende da abertura delas, como também da frequência de rotação das bombas. São 4 ao todo, uma na entrada de cada tanque.

O primeiro procedimento consistiu em, para uma posição fixa de cada válvula de entrada, variar a frequência da rotação da bomba e calcular a vazão obtida através da contagem do tempo necessário para o preenchimento do volume total do tanque $(100 \%$, equivalente a 11L). Feito isso, elaborou-se funções que representavam a relação Vazão $x$ Frequência (que se mostrou linear) para cada tanque. A partir dos resultados obtidos, foi possível utilizar a vazão como variável manipulada do processo.

Tem-se também válvulas de saída dos tanques, cujas vazões dependem da abertura das mesmas e da altura manométrica dos tanques.

O segundo procedimento consistiu em, para uma posição fixa de cada válvula de saída, medir várias vazões de entrada e seus respectivos níveis estacionários. Nesta situação, sabe-se que a vazão de entrada é igual à vazão de saída, logo tem-se a relação entre h (altura do fluido no tanque) e Qsaída (Vazão de saída), de forma a se encontrar a resistência que a válvula impõe ao fluxo.

Assim, deve-se perceber e entender que todas as válvulas foram definidas em uma abertura fixa (resistência constante ao fluxo), de modo a se ter sempre uma mesma relação entre frequência de rotação da bomba e vazão ou altura manométrica e a vazão.

\section{MODELAGEM DO SISTEMA}

A modelagem deste processo envolve apenas balanço de massa, já que não há variações apreciáveis de temperatura.

Existem muitos modelos possíveis, mas, optou-se por não utilizar o sistema de tanques comunicantes, como também optou-se por impor que todo o fluido que for direcionado aos tanques superiores ( 1 e 2 ) necessariamente escoe para os tanques inferiores (3 e 4, respectivamente), para só então seguir para o reservatório. 
Supõe-se que as válvulas utilizadas apresentam comportamento não linear. Portanto, usou-se o modelo mais utilizado pela literatura para relação não linear entre vazão de saída e nível em tanques:

$$
Q_{s}=\beta \sqrt{h}
$$

Assim sendo, o modelo proposto torna-se:

Tanque 1: $\quad Q_{E, 1}-\beta_{1} \sqrt{h_{1}}=\frac{d h_{1}}{d t}$

Tanque 2: $\quad Q_{E, 2}-\beta_{2} \sqrt{h_{2}}=\frac{d h_{2}}{d t}$

Tanque 3: $\quad Q_{E, 3}+\beta_{1} \sqrt{h_{1}}-\beta_{3} \sqrt{h_{3}}=\frac{d h_{3}}{d t}$

Tanque 4: $\quad Q_{E, 4}+\beta_{2} \sqrt{h_{2}}-\beta_{4} \sqrt{h_{4}}=\frac{d h_{4}}{d t}$

Sendo: $Q_{E, i}=$ vazão associada à válvula de entrada independente do tanque $\mathrm{i}$;

$$
\begin{aligned}
& \beta_{i}=\text { parâmetro associado à resistência da válvula de saída do tanque } \mathrm{i} ; \\
& h_{i}=\text { nível do tanque } \mathrm{i}
\end{aligned}
$$

O modelo depende da adequação destas equações ao protótipo experimental. Com este objetivo, fez-se as curvas de Q $\mathrm{x}$ h para os 4 tanques, no estado estacionário. Para os dois tanques inferiores (3 e 4), fez-se também a restrição de que as únicas vazões de entrada fossem aquelas vindas das "válvulas de entrada independente", já especificadas acima. Assim, para o procedimento realizado, a equação para todos os tanque é a seguinte:

Tanque i

$$
Q_{E, i}=\beta_{i} \sqrt{h_{i}}
$$

\section{RESULTADOS}

\subsection{Determinação das Vazões do Sistema}

As funções para a determinação das vazões de entrada dos tanques do sistema foram do tipo linear e com coeficientes de correlação satisfatórios para o ajuste. As funções são apresentadas abaixo:

Vazão de entrada do Tanque 1:

$$
\begin{array}{ll}
\mathrm{Q}=0,0173 * \mathrm{P}-0,5354 & \mathrm{R}^{2}=0,9747 \\
\mathrm{Q}=0,0184 * \mathrm{P}-0,5699 & \mathrm{R}^{2}=0,9764 \\
\mathrm{Q}=0,0151 * \mathrm{P}-0,3121 & \mathrm{R}^{2}=0,9846 \\
\mathrm{Q}=0,0116^{*} \mathrm{P}-0,1993 & \mathrm{R}^{2}=0,9902
\end{array}
$$

Vazão de entrada do Tanque 2:

Vazão de entrada do Tanque 3:

Vazão de entrada do Tanque 4: 
Sendo que: - Vazão Q, em L/s; - Frequência de Rotação da Bomba P, em percentual da frequência máxima da bomba $(60 \mathrm{~Hz})$.

\section{2 . Determinação dos Parâmetros dos Modelos Não Lineares das Válvulas.}

As equações representadas pela equação 6 foram ajustadas aos dados experimentais em diferentes regimes permanentes. As curvas de Q x h são apresentadas nas figuras 3, 4, 5 e 6.

Figura 3: Curva de Q x h para o Tanque 1

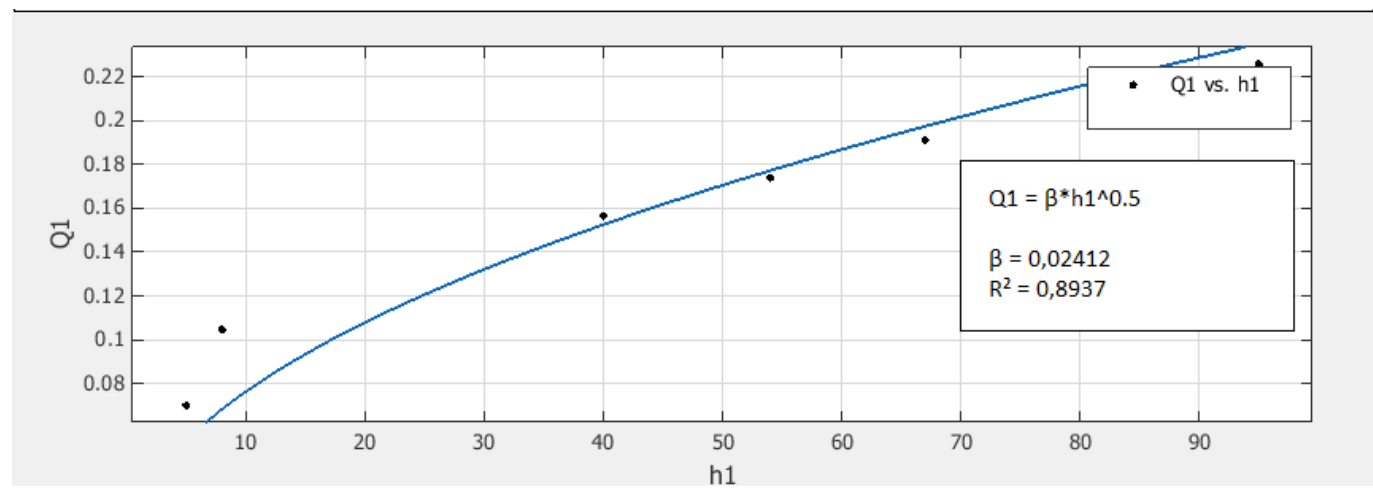

Figura 4: Curva de Q x h para o Tanque 2

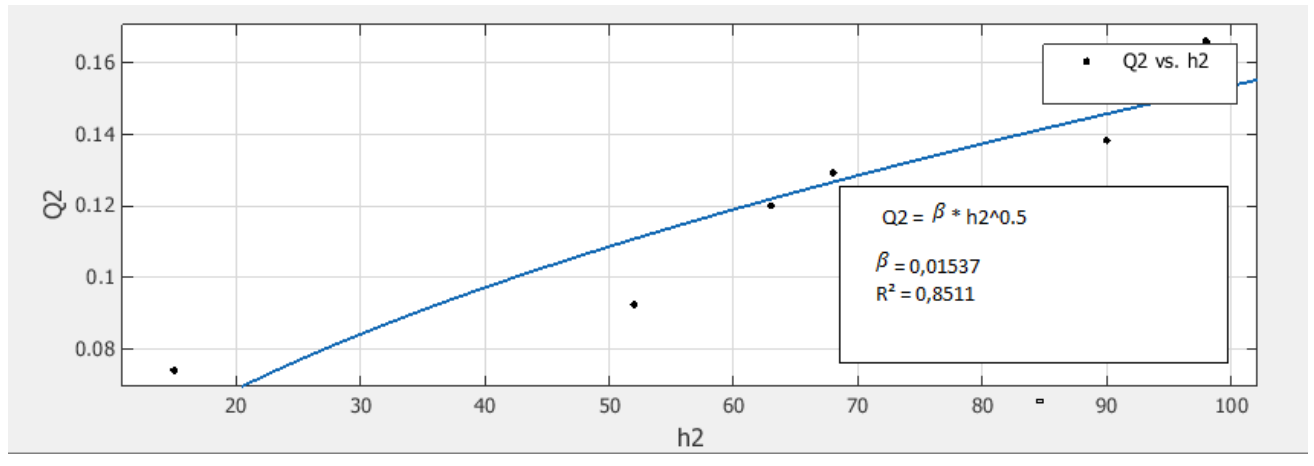

Figura 5: Curva de Q x h para o Tanque 3

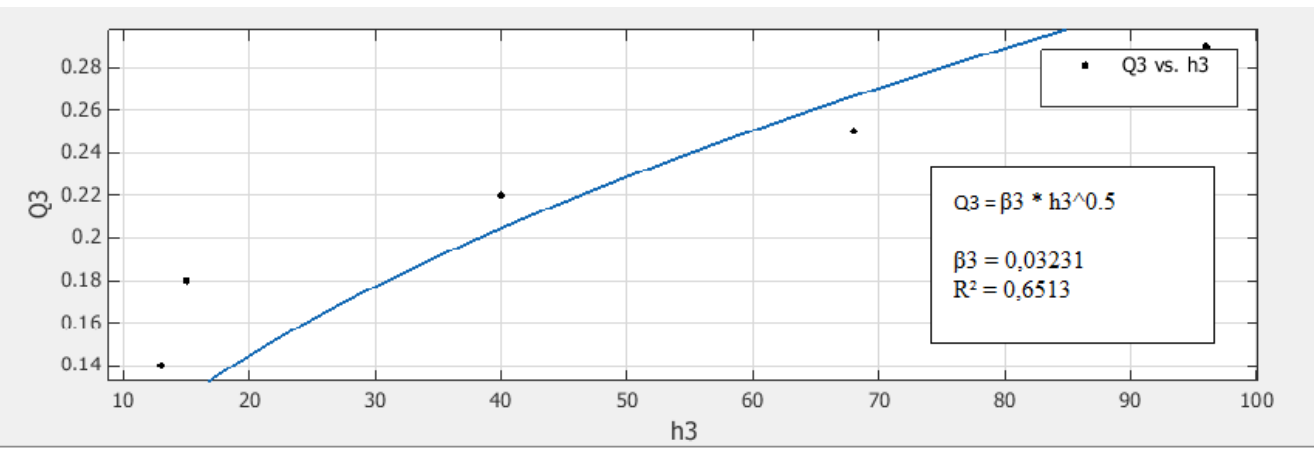


Figura 6: Curva de Q x h para o Tanque 4

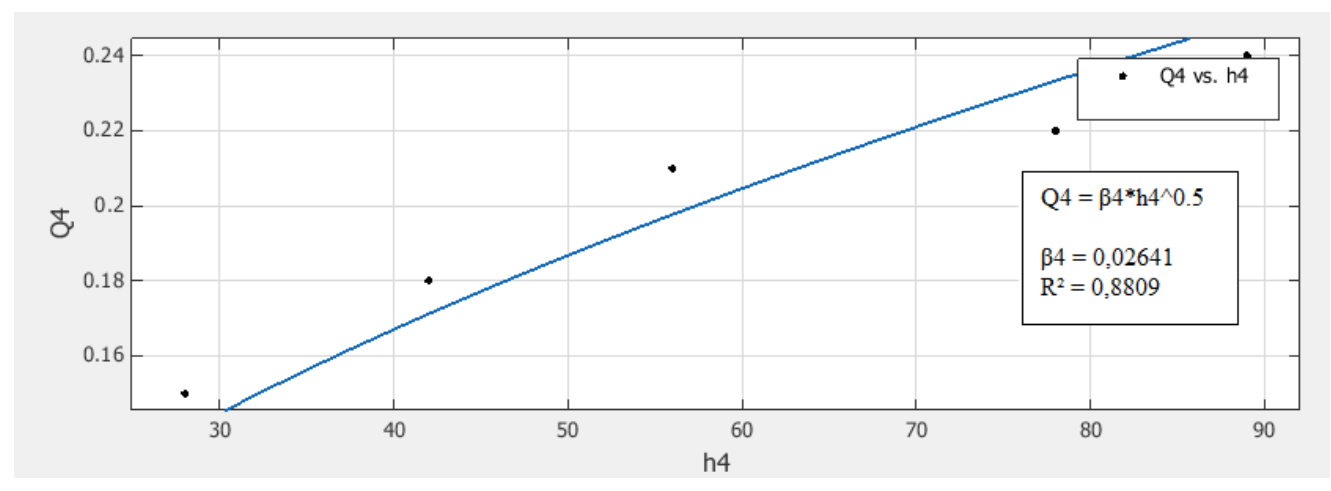

Os valores dos parâmetros $\beta$ das funções não lineares das válvulas foram ajustados. Estes valores representam a resistência que a válvula impõe ao escoamento. Os valores de $\mathrm{R}^{2}$ encontrados não foram satisfatórios, indicando a necessidade de mais dados experimentais para melhorar o ajuste. Entretanto, eles podem ser considerados aceitáveis, uma vez que um sistema deste representa fortemente a realidade de uma indústria, por possuir muitos problemas operacionais. Pode-se citar a forte turbulência nos tanques, que gera uma alta imprecisão no sensor de nível e o superdimensionamento da bomba, que restringe seu uso a uma faixa percentual pequena do seu valor. Estes problemas serão sanados ao longo do trabalho de Iniciação Científica. Ressalta-se que este trabalho de Iniciação Científica está em fase inicial e que este modelagem será fundamental para a definição dos controladores baseados em Inteligência Artificial.

\section{CONCLUSÃO}

Testes na planta possibilitaram a modelagem empírica do sistema. Diversos problemas operacionais foram detectados e serão paulatinamente resolvidos. Esse projeto possibilitará uma aplicação prática dos conceitos teóricos de controle e instrumentação de processos, permitindo uma familiarização com controladores lógicos programáveis e softwares de simulação.

\section{REFERÊNCIAS BIBLIOGRÁFICAS}

Fernandez, J. D.; Fernandez, A. E.; SCADA systems: vulnerabilities and remediation, Journal of Computing Sciences in Colleges, Vol 20, Texas, USA, p. 160-168, 2005.

Johansson, K.H., Relay Feedback and Multivariable Control, Tese de Doutorado, Lund Institute of Technology, Sweden, 162p., 1997.

Johansson, K.H., Horcht,A., Wijkt, O., Hanssont, A., Teaching Multivariable Control Using the Quadruple-Tank Process, Proceedings of the 38th Conference on Decision \& Control, Arizona, USA, 1999.

Seborg, D. E.; Edgar, T F.; Mellichamp, D. A.; Process Dynamics and Control, 2a Edition, John Wiley \& Sons, New York, 2004 